\title{
Fluorodeoxyglucose-positron emission tomogra- phy/computed tomography imaging of squamous cell carcinoma arising in a meningomyelocele
}

\author{
Seval Erhamamc1 ${ }^{1}$, Mehmet Reyhan ${ }^{1}$, and Nebil Bal ${ }^{2}$
}

Departments of ${ }^{1}$ Nuclear Medicine and ${ }^{2}$ Pathology, Baskent University Faculty of Medicine, Ankara, Turkey

Received: March 24, 2015

Revised : May 5, 2015

Accepted: May 12, 2015

\section{Correspondence to}

\section{Seval Erhamamc1, M.D.}

Tel: +90-332-257-0606

Fax: +90-332-257-0637

E-mail: sevaler@yahoo.com
The patient was a 26-years-old female with a history of lumbosacral meningomyelocele with paraplegia. She was admitted to the hospital due to the lumbosacral wound having become infecting and producing a discharge for 6-months. The patient underwent excisional biopsy of the lumbosacral region. The pathological diagnosis was consistent with metastatic moderately-differentiated squamous cell carcinoma (SCC) (Fig. 1). Fluorodeoxyglucose-positron emission tomography/ computed tomography (FDG-PET/CT) imaging was performed for the detection and localisation of the primary tumor and metastatic evaluation (Fig. 2).

The development of carcinoma associated with meningomyelocele is rare and only limited sporadic cases have been reported in the literature. Report-

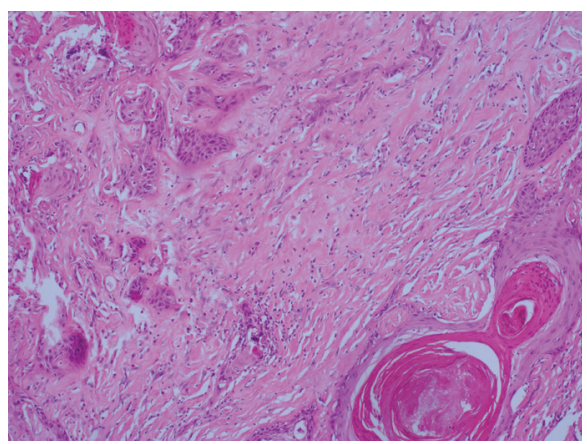

Figure 1. Atypical squamous cells have keratinization that infiltrating desmoblastic stroma (H\&E, ×100). ed cases were usually of SCC, as in our case. It has been proposed that malignancy arises at these sites as a result of chronic mechanical irritation and chronic infection, again as in our case. The term Marjolin's ulcer is often used to describe the formation of neoplastic changes in the scar tissue of chronic ulcers. The most common cell type is SCC, and those SCCs resulting from Marjolin's ulcers have a much greater tendency to metastasize than those SCCs arising from other causes, which makes early diagnosis imperative.

Imaging studies are useful for the characterization of the tumor, the evaluation of its extent, and are essential for treatment planning. FDG-PET/CT whole-body imaging is a widely used technique for the evaluation of many types of malignancies including SCC and is particularly useful for the detection of the primary tumor of unknown origin. FDG-PET/CT can also identify additional sites of metastases that can alter the patient's management. In a recent study, PET/CT was shown to be useful in differentiating Marjolin ulcer from benign inflammatory conditions of chronic nonhealing ulcer in burn scars and in the evaluation of the depth of invasion in Marjolin's ulcer cases. 

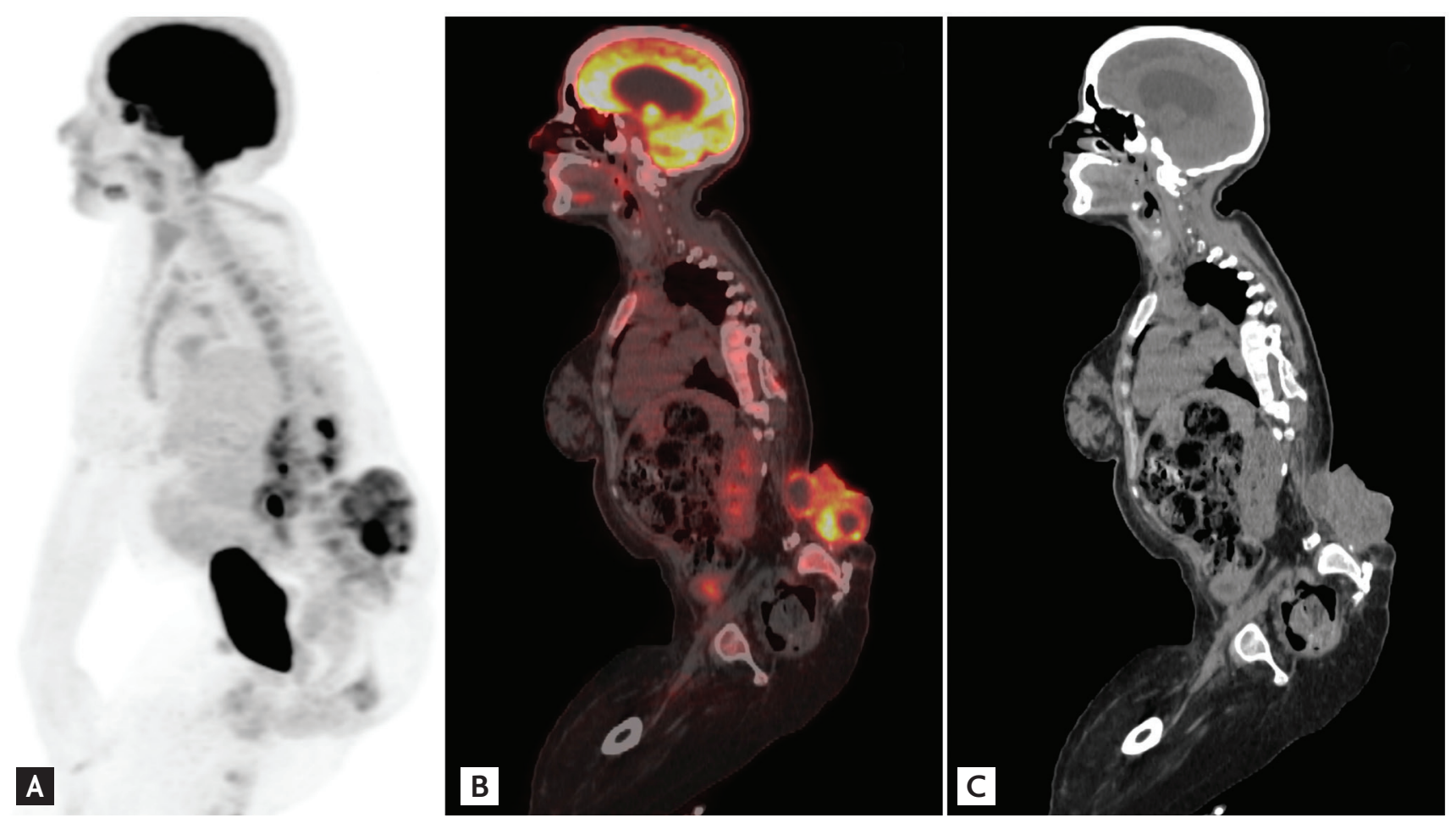

Figure 2. (A) Maximum intensity projection, (B) sagittal fusion positron emission tomography/computed tomography (PET/CT), and (C) CT images show heterogeneously increased high fluorodeoxyglucose (FDG) uptake (maximum standardized uptake value [SUVmax], 12.7) including hypometabolic area within the lesion in the lumbosacral region due to squamous cell carcinoma, and mild FDG uptake within right axillary (SUVmax, 2.6) and bilateral inguinal (SUVmax, 3.4) lymph nodes.

\section{Conflict of interest}

No potential conflict of interest relevant to this article was reported. 